\title{
Internal Quadratic Invariance and Decentralized Control
}

\author{
Laurent Lessard $^{1} \quad$ Sanjay Lall ${ }^{2}$ \\ American Control Conference, pp. 5596-5601, 2010
}

\begin{abstract}
For decentralized control systems with quadratically invariant information constraints, the set of achievable closed-loop maps is affine, and thus the associated minimum-norm controller synthesis problem is amenable to a convex programming approach. In this paper, we show that a strictly broader class of problems we call internally quadratically invariant, also yields an affine set of achievable closed-loop maps. We treat systems represented by rational as well as proper rational transfer functions and present an illustrative example.
\end{abstract}

\section{Introduction}

In decentralized control problems, one has multiple controllers, each of which has access to different measurement information. Such situations arise often in large systems, where it is impossible or infeasible to implement a centralized controller. For example, packet routing in networks requires each hub to make decisions regarding its local routing policy without knowledge of the full state of the network.

The decentralization requirement can be expressed as a constraint on the linear operator $K$ representing the controller, and for many decentralized systems this constraint takes the form that $K$ must lie in some subspace $S$. Examples include sparsity constraints where each controller has access to different measurement channels, as well as delay constraints.

Recent approaches to optimal decentralized control have made use of the linear-fractional framework. In this representation, we would like to solve

$$
\begin{aligned}
\operatorname{minimize} & \left\|P_{11}+P_{12} K(I-G K)^{-1} P_{21}\right\| \\
\text { subject to } & K \in S \\
& K \text { is stabilizing }
\end{aligned}
$$

where $\|\cdot\|$ is any system norm that captures the control performance objectives. A property called quadratic invariance, developed in [5], offers an approach for solving

\footnotetext{
${ }^{1}$ L. Lessard is with the Department of Aeronautics and Astronautics at Stanford University, Stanford, CA 94305, USA. lessard@stanford.edu

${ }^{2} \mathrm{~S}$. Lall is with the Department of Electrical Engineering and Aeronautics and Astronautics at Stanford University, Stanford, CA 94305, USA. lall@stanford.edu

${ }^{3}$ This work was supported by NSF grant number 0642839
}

a wide class of such problems. Define for convenience the function $h(K)=-K(I-G K)^{-1}$, which is the underlying map in the well-known Youla parametrization.

The main object of interest here is the set of achievable closed-loop maps; the quantity inside the norm of the optimization problem (1). If we substitute the definition for $h$, we can express this set more compactly as

$$
P_{11}-P_{12} h(S) P_{21} .
$$

If $S$ is quadratically invariant with respect to $P_{22}$, then subject to some additional technical conditions, we have $h(S)=S$. This reduces (1) to

$$
\begin{aligned}
\operatorname{minimize} & \left\|P_{11}-P_{12} Q P_{21}\right\| \\
\text { subject to } & Q \in S \\
& Q \text { is stable, }
\end{aligned}
$$

which has the advantage of being convex in $Q$. See [6] for details. The key property here is that makes the optimization problem tractable is that the set of achievable closed-loop maps is affine. However, quadratic invariance is only a sufficient condition. it can happen that the set

$$
P_{11}-P_{12} h(S) P_{21}
$$

is affine, even though $h(S)$ is not a subspace. In this case, finding the best achievable control performance is no more difficult, even though the set $S$ is not quadratically invariant. Our purpose in this paper is to extend the notion of quadratic invariance to capture such cases. In particular, we define internal quadratic invariance of $S$ with respect to the plant $P$. When this property holds, we have

$$
P_{12} h(S) P_{21}=P_{12} S P_{21}
$$

even though $h(S)$ may not equal $S$. The corresponding results in this paper are Theorem 5 (for rational functions), and Theorem 7 (for proper rationals).

The proper rational case is of practical interest because it represents transfer functions that can be realized using circuits or computers. Proper transfer functions in discrete-time represent causal systems, and in continuous-time represent systems with no pure differentiators. Finally in Section 5, we show an example.

Prior work. A rich theory exists surrounding the solution of centralized control problems. In many cases, the optimal controller is unique and efficient methods exist for computing it. See for example [1]. 
Due to the advent of increasingly complex systems, there has been growing interest in large-scale and decentralized control problems. However, even simple tractable centralized problems can become intractable when a decentralized constraint is imposed [8].

The tractable decentralized control problems have not been completely characterized, but large classes of tractable problems are known. For example, Ho and Chu [2] identified the partially nested systems, for which optimal controllers can be easily computed. It was later shown that such systems belong to an even larger class, named quadratically invariant (QI) $[5,6]$. In these systems, the set of achievable closed-loop maps is affine, which makes the problem amenable to convex search. Recently, this class was extended further [4], by showing that some non-QI systems can be transformed into QI systems via a change of variables.

\section{Preliminaries}

We review some properties of matrices of rational functions. Define $\mathcal{R}^{m \times n}, \mathcal{R}_{p}^{m \times n}$, and $\mathcal{R}_{s p}^{m \times n}$ to be the set of $m \times n$ matrices in which each entry is a real-rational, proper real-rational, and strictly proper real rational function, respectively. We omit the superscript when the matrix dimensions are to be inferred by context.

Note that the sets $\mathcal{R}, \mathcal{R}_{p}, \mathcal{R}_{s p}$ are vector spaces using the field $\mathbb{R}$ for scalar multiplication. If $S \subset \mathcal{R}$ is a subspace, and $r K \in S$ for all $r \in \mathcal{R}$ and $K \in S$, we call $S$ an $\mathcal{R}$-subspace. Note that $\mathcal{R}$ is an $\mathcal{R}$-subspace, but $\mathcal{R}_{p}$ and $\mathcal{R}_{s p}$ are not.

If $A \in \mathcal{R}^{m \times n}$, define its range and nullspace as

$$
\begin{aligned}
\operatorname{range} A & =\left\{A x \mid x \in \mathcal{R}^{n}\right\} \\
\text { null } A & =\left\{x \in \mathcal{R}^{n} \mid A x=0\right\} .
\end{aligned}
$$

Both these sets are $\mathcal{R}$-subspaces.

Suppose $A \in \mathcal{R}^{n \times n}$. We say that $A$ is invertible if its determinant $\operatorname{det} A(s)$ is not identically zero. Note that we do not require $A$ or its inverse to be proper. We call $A$ a projector if $A=A^{2}$.

The plant $P \in \mathcal{R}^{m \times n}$ is partitioned as

$$
\left[\begin{array}{l}
z \\
y
\end{array}\right]=\left[\begin{array}{ll}
P_{11} & P_{12} \\
P_{21} & P_{22}
\end{array}\right]\left[\begin{array}{l}
w \\
u
\end{array}\right],
$$

with $m=m_{1}+m_{2}$ and $n=n_{1}+n_{2}$, and $P_{11} \in \mathcal{R}^{m_{1} \times n_{1}}$, $P_{12} \in \mathcal{R}^{m_{1} \times n_{2}}, P_{21} \in \mathcal{R}^{m_{2} \times n_{1}}$, and $P_{22} \in \mathcal{R}^{m_{2} \times n_{2}}$. The controller $K \in \mathcal{R}^{n_{2} \times m_{2}}$ is connected via $u=K y$, and these equations are illustrated in the block diagram of Figure 1.

We will use the convention that $G=P_{22}$ throughout this paper. Define the set

$$
M=\left\{K \in \mathcal{R}^{n_{2} \times m_{2}} \mid(I-G K) \text { is invertible }\right\}
$$

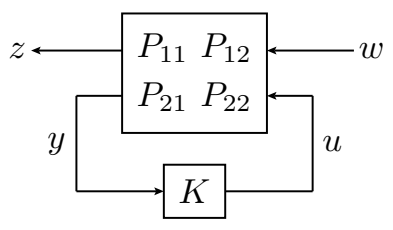

Figure 1: Closed-loop interconnection

and the function $h: M \rightarrow M, h(K)=-K(I-G K)^{-1}$. Whenever $K \in M$, we say the interconnection of Figure 1 is well-posed, and the closed-loop map is

$$
f(P, K)=P_{11}-P_{12} h(K) P_{21} .
$$

In this paper, we will consider sets of interconnections parametrized by $K \in S$. We call the subspace $S \subset \mathcal{R}^{n_{2} \times m_{2}}$ the information constraint.

Quadratic Invariance. We say $S$ is quadratically invariant with respect to $G$ if $K G K \in S$ whenever $K \in S$. Under suitable technical conditions, quadratic invariance is necessary and sufficient for the constraint $S$ to be preserved under $h$. In other words, $h(S)=S$, and consequently the set of achievable closed-loop maps is affine. One such result is Lemma 10. Similar results exist for proper rationals [5], Banach spaces [5], and more general topological spaces [6].

\section{Rational Functions}

We will require that the information constraint $S$ be an $\mathcal{R}$-subspace for all the results in this paper. This class is quite general, and includes sparsity constraints, as well as frequency-aligned constraints [5]. Refer to Lemma 9 for a useful characterization of $\mathcal{R}$-subspaces.

Our main result is that subject to a condition we call internal quadratic invariance, the set of achievable closed-loop maps is affine, and thus amenable to convex search.

Definition 1. Suppose $P \in \mathcal{R}^{m \times n}$, and $S \subset \mathcal{R}^{n_{2} \times m_{2}}$ is an $\mathcal{R}$-subspace. Let $W_{1}$ and $W_{2}$ be projectors such that

$$
\begin{aligned}
\text { range } W_{1} & =\text { range }\left[\begin{array}{ll}
P_{21} & P_{22}
\end{array}\right] \\
\text { null } W_{2} & =\text { null }\left[\begin{array}{l}
P_{12} \\
P_{22}
\end{array}\right]
\end{aligned}
$$

We say $S$ is internally quadratically invariant (IQI) with respect to $P$ if $W_{2} S W_{1}$ is $Q I$ with respect to $G=P_{22}$.

We will now show that internal quadratic invariance does not depend on the choice of projectors $W_{1}$ and $W_{2}$, it is a property of $P$ and $S$ alone. First, we show the required existence property, that it is always possible to construct projectors satisfying (2). 
Lemma 2. Suppose $A \in \mathcal{R}^{m \times n}$. There exists a proper projector $W \in \mathcal{R}_{p}^{m \times m}$ (or $W \in \mathcal{R}_{p}^{n \times n}$ ) with the same range (or nullspace) as $A$.

Proof. Write $A$ as

$$
A=\underbrace{\left[\begin{array}{ll}
U_{1} & U_{2}
\end{array}\right]}_{U} \underbrace{\left[\begin{array}{cc}
M_{1} & 0 \\
0 & 0
\end{array}\right]}_{M} \underbrace{\left[\begin{array}{c}
V_{1}^{T} \\
V_{2}^{T}
\end{array}\right]}_{V}
$$

where $U$ and $V$ are unimodular polynomial matrices and $M$ is the Smith-McMillan form of $A$ [3]. Then, it is straightforward to verify that $W_{1}=U_{1}\left(U_{1}^{T} U_{1}\right)^{-1} U_{1}^{T}$ is a projector with range $W_{1}=$ range $A$. Similarly, $W_{2}=$ $V_{1}\left(V_{1}^{T} V_{1}\right)^{-1} V_{1}^{T}$ is a projector with null $W_{2}=$ null $A$.

One can show that the $\operatorname{limit} \lim _{\alpha \rightarrow \infty} W(\alpha)$ is always a constant for projectors constructed in this fashion, and thus they are proper.

Next, we show that for fixed $P$ and $S$, whether or not $W_{2} S W_{1}$ is QI with respect to $G$ does not depend on our choice of projectors.

Lemma 3. Suppose $P \in \mathcal{R}^{m \times n}$, and $S \subset \mathcal{R}^{n_{2} \times m_{2}}$ is an $\mathcal{R}$-subspace. Suppose further that $W_{i}$ and $Z_{i}$ are two sets of projectors satisfying (2). Then the two following statements are equivalent

i) $W_{2} S W_{1}$ is $Q I$ with respect to $G$

ii) $Z_{2} S Z_{1}$ is $Q I$ with respect to $G$

Proof. Since $W_{1}$ and $Z_{1}$ have the same range, each column of $Z_{1}$ is a linear combination of the columns of $W_{1}$. Therefore we can write $Z_{1}=W_{1} X$ for some $X \in$ $\mathcal{R}^{m_{2} \times m_{2}}$. Similar arguments imply that $W_{2}^{T}$ and $Z_{2}^{T}$ have the same range, so we may similarly conclude that $Z_{2}=Y W_{2}$ for some $Y \in \mathcal{R}^{n_{2} \times n_{2}}$.

Now suppose that $W_{2} S W_{1}$ is QI with respect to $G$. Then for all $K \in S$,

$$
\left(W_{2} K W_{1}\right) G\left(W_{2} K W_{1}\right) \in W_{2} S W_{1}
$$

Multiply on the left by $Y$ and on the right by $X$, and deduce that

$$
\left(Z_{2} K W_{1}\right) G\left(W_{2} K Z_{1}\right) \in Z_{2} S Z_{1}
$$

From Lemma 13, we have

$$
W_{1} G W_{2}=Z_{1} G Z_{2}=G
$$

and so

$$
\left(Z_{2} K Z_{1}\right) G\left(Z_{2} K Z_{1}\right) \in Z_{2} S Z_{1}
$$

and we conclude that $Z_{2} S Z_{1}$ is QI with respect to $G$. The same argument holds if we interchange $W$ and $Z$, and this completes the proof.

Quadratic invariance is only a property of the information constraint $S$ and the $P_{22}$ block of the plant. However, internal quadratic invariance also depends on the other blocks $P_{i j}$ of the plant. We now show that IQI is weaker than QI. That is, all QI systems are IQI.
Theorem 4. If $S$ is $Q I$ with respect to $G$. Then $S$ is IQI with respect to $P$.

Proof. Choose $W_{1}$ and $W_{2}$ such that (2) holds. Suppose $S$ is QI with respect to $G$. Then for any $K \in S$, $K G K \in S$, and thus $W_{2} K G K W_{1} \in W_{2} S W_{1}$. We also have by Lemma 13 that $W_{1} G W_{2}=G$. Therefore $\left(W_{2} K W_{1}\right) G\left(W_{2} G W_{1}\right) \in W_{2} S W_{1}$.

Most notably, if $\left[\begin{array}{ll}P_{21} & P_{22}\end{array}\right]$ and $\left[\begin{array}{l}P_{12} \\ P_{22}\end{array}\right]$ have full normal rank, both projectors $W_{1}$ and $W_{2}$ as defined in (2) are the identity. In this case, the two notions are equivalent: $S$ is IQI with respect to $P$ if and only if $S$ is QI with respect to $G$.

In general, an IQI system may not be QI. In Section 5, we give an example of such a system. Here is a statement of the main result of this section.

Theorem 5. Suppose $P \in \mathcal{R}^{m \times n}, S \subset \mathcal{R}^{n_{2} \times m_{2}}$ is an $\mathcal{R}$-subspace, and $S$ is IQI with respect to $P$. Then

$$
P_{12} h(S \cap M) P_{21}=P_{12}(S \cap M) P_{21}
$$

\section{Proof. See Section 6}

An immediate consequence of Theorem 5 is that when $S$ is IQI with respect to $P$, the set of achievable closedloop maps may be written as

$$
\begin{aligned}
f(P, S \cap M) & =P_{11}-P_{12} h(S \cap M) P_{21} \\
& =P_{11}-P_{12}(S \cap M) P_{21}
\end{aligned}
$$

which is an affine set. This allows us to transform a broad class of decentralized optimization problems into convex programs. For example, if we are interested in solving an optimization problem of the form

$$
\begin{aligned}
\operatorname{minimize} & \|f(P, K)\| \\
\text { subject to } & K \in S \cap M
\end{aligned}
$$

then we need only find the $Q^{*} \in S$ that minimizes $\left\|P_{11}-P_{12} Q P_{21}\right\|$, and then the solution to (3) is related via the equation $P_{12} h\left(K^{*}\right) P_{21}=P_{12} Q^{*} P_{21}$.

To find $K^{*}$ explicitly, we apply the proof technique from Lemma 11. Namely, we use the fact that $h$ is involutive to conclude that $W_{2} K^{*} W_{1}=h\left(W_{2} Q^{*} W_{1}\right)$. This is now a linear equation for $K^{*}$, where $K^{*}$ is constrained to lie in the subspace $S$.

Note that the norm used in (3) may be any system norm. For example, the $\mathcal{H}_{\infty}$-norm or the $\mathcal{H}_{2}$-norm.

\section{Proper Rationals}

In this section, we treat the case where the plant is strictly proper and the controller is proper. Throughout this section, we will use the notation $S_{p}=S \cap \mathcal{R}_{p}$ for brevity. 
This case is fundamentally different from the general rational case because although $\mathcal{R}_{p}$ is a subspace over the complex field, it is not an $\mathcal{R}$-subspace. So Theorem 5 does not apply. Indeed, Lemma 8, which was used to prove Theorem 5, fails to hold. For a simple counterexample, consider solving the linear equation $W x=b$ subject to the constraint $x \in S$, where

$$
W=\frac{1}{s^{2}+1}\left[\begin{array}{cc}
1 & s \\
s & s^{2}
\end{array}\right], \quad b=\frac{1}{s}\left[\begin{array}{l}
1 \\
s
\end{array}\right], \quad S=\left[\begin{array}{c}
\mathcal{R} \\
0
\end{array}\right]
$$

Despite $W$ and $b$ being proper, the only solution is $x=$ $\left[\begin{array}{c}\frac{s^{2}+1}{s} \\ 0\end{array}\right]$, which is not proper. In other words, the system has no solution in $S_{p}$, although it does have solutions in $S$ and in $\mathcal{R}_{p}$.

In order to conclude something definitive about the achievable closed-loop maps, we will make a technical assumption called proper-preserving. This notion is also used in [7].

Definition 6. We say $(P, S)$ is proper-preserving if $K$ is proper whenever $P_{12} h(K) P_{21}$ is proper.

The main result of this section is as follows.

Theorem 7. Suppose $P \in \mathcal{R}_{s p}^{m \times n}, S \subset \mathcal{R}^{n_{2} \times m_{2}}$ is an $\mathcal{R}$ subspace, $(P, S)$ is proper-preserving, and $S$ is IQI with respect to $P$. Then

$$
P_{12} h\left(S_{p}\right) P_{21}=P_{12} S P_{21} \cap \mathcal{R}_{p}^{m_{1} \times n_{1}}
$$

\section{Proof. See Section 6}

We may use Theorem 7 to reduce certain optimization problems to convex programs, as we did in Section 3. Suppose we have a strictly proper plant $P$, and we would like to find a proper controller that minimizes a norm of the closed-loop system:

$$
\begin{aligned}
\text { minimize } & \|f(P, K)\| \\
\text { subject to } & K \in S_{p}
\end{aligned}
$$

If $(P, S)$ is proper-preserving, Theorem 7 tells us that $P_{12} h\left(S_{p}\right) P_{21}=P_{12} S P_{21} \cap \mathcal{R}_{p}^{m_{1} \times n_{1}}$. Therefore, if $Q^{*}$ is the optimal solution to

$$
\begin{aligned}
\operatorname{minimize} & \left\|P_{11}-P_{12} Q P_{21}\right\| \\
\text { subject to } & Q \in S \text { and } \\
& P_{12} Q P_{21} \text { is proper }
\end{aligned}
$$

then $P_{12} Q^{*} P_{21} \in P_{12} h\left(S_{p}\right) P_{21}$. And so we can find $K^{*} \in$ $S_{p}$ that satisfies $P_{12} Q^{*} P_{21}=P_{12} h\left(K^{*}\right) P_{21}$, and $K^{*}$ will be the optimal solution to (4).

\section{Example}

We now show an example of an internally QI system. Consider the following plant and information constraint.

$$
\begin{aligned}
& P=\left[\begin{array}{c:ccc}
a & b_{1} & b_{2} & b_{2} \\
\hdashline c_{1} & g_{1} & 0 & 0 \\
c_{1} & g_{1} & 0 & 0 \\
c_{2} & g_{2} & g_{3} & g_{3}
\end{array}\right] \\
& S=\left\{\left[\begin{array}{ccc}
k_{1} & 0 & 0 \\
0 & k_{2} & 0 \\
0 & 0 & k_{3}
\end{array}\right] \mid k_{i} \in \mathcal{R}\right\}
\end{aligned}
$$

where $a, b_{i}, c_{i}$, and $g_{i}$ are rational functions. Note that the dashed lines are used to partition $P$ into its $P_{i j}$ blocks and this does not denote a state-space representation.

It is clear that $S$ is not QI with respect to $P_{22}$, since $K P_{22} K$ is not generally diagonal for diagonal $K$. Now compute projectors as in Definition 1.

$$
\begin{array}{r}
\text { range }\left[\begin{array}{cccc}
c_{1} & g_{1} & 0 & 0 \\
c_{1} & g_{1} & 0 & 0 \\
c_{2} & g_{2} & g_{3} & g_{3}
\end{array}\right]=\text { range } \underbrace{\left[\begin{array}{ccc}
\frac{1}{2} & \frac{1}{2} & 0 \\
\frac{1}{2} & \frac{1}{2} & 0 \\
0 & 0 & 1
\end{array}\right]}_{W_{1}} \\
\text { null }\left[\begin{array}{ccc}
b_{1} & b_{2} & b_{2} \\
g_{1} & 0 & 0 \\
g_{1} & 0 & 0 \\
g_{2} & g_{3} & g_{3}
\end{array}\right]=\text { null } \underbrace{\left[\begin{array}{cccc}
1 & 0 & 0 \\
0 & \frac{1}{2} & \frac{1}{2} \\
0 & \frac{1}{2} & \frac{1}{2}
\end{array}\right]}_{W_{2}}
\end{array}
$$

Now compute the set $W_{2} S W_{1}$

$$
W_{2} S W_{1}=\left\{\left[\begin{array}{ccc}
k_{1} & k_{1} & 0 \\
k_{2} & k_{2} & k_{3} \\
k_{2} & k_{2} & k_{3}
\end{array}\right] \mid k_{i} \in \mathcal{R}\right\}
$$

and notice that

$$
\left(W_{2} K W_{1}\right) G\left(W_{2} K W_{1}\right)=\left[\begin{array}{ccc}
v_{1} & v_{1} & 0 \\
v_{2} & v_{2} & v_{3} \\
v_{2} & v_{2} & v_{3}
\end{array}\right] \in W_{2} S W_{1}
$$

where $v_{1}=\frac{1}{2} g_{1} k_{1}^{2}, v_{2}=\frac{1}{4}\left(g_{1} k_{1} k_{2}+g_{2} k_{1} k_{3}+g_{3} k_{2} k_{3}\right)$, and $v_{3}=\frac{1}{2} g_{3} k_{3}^{2}$. Therefore $W_{2} S W_{1}$ is QI with respect to $G$, and so $S$ is internally quadratically invariant with respect to $P$.

Applying Theorem 5, we have that $P_{12} h(S) P_{21}$ is an affine set, and so we may use convex programming to solve an associated control synthesis problem. Note that $h(S)$ is not affine in this case, because $S$ being QI with respect to $P_{22}$ is both necessary and sufficient for $h(S)$ to be affine.

\section{Proofs}

In this section, we will give proofs of Theorems 5 and 7 . Before doing so, we require some lemmas. 
Lemma 8. Suppose $A \in \mathcal{R}^{m \times n}$ and $b \in \mathcal{R}^{m}$. Further suppose that for all $\alpha \in \mathbb{R}$ at which both $A$ and $b$ are defined, there exists a vector of real numbers $z \in \mathbb{R}^{n}$ that satisfies the equation $A(\alpha) z=b(\alpha)$. Then there exists some $x \in \mathcal{R}^{n}$ such that $A x=b$.

Proof. This result follows by constructing $x$ using Gaussian elimination over the field of rational functions. We omit the details because of space constraints.

Lemma 9. Suppose $S \subset \mathcal{R}^{m \times n}$. Then $S$ is an $\mathcal{R}$ subspace if and only if for all $\alpha \in \mathbb{R}$, there exists a subspace $S_{\alpha} \subset \mathbb{R}^{m \times n}$ such that

$$
S=\left\{K \in \mathcal{R}^{m \times n} \mid K(\alpha) \in S_{\alpha} \text { for almost all } \alpha \in \mathbb{R}\right\}
$$

Proof. Suppose $S$ is defined as in Lemma 9. Now pick any $K \in S$ and $r \in \mathcal{R}$. For almost all $\alpha \in \mathbb{R}, K(\alpha) \in S_{\alpha}$. Since $S_{\alpha}$ is a subspace, we also have $r(\alpha) K(\alpha) \in S_{\alpha}$. It follows that $r K \in S$.

Conversely, suppose that $r K \in S$ for all $K \in S$ and $r \in$ $\mathcal{R}$. This means $S$ is a vector field where the scalars are the field of rational functions $\mathcal{R}$. $S$ has finite dimension, since $S \subset \mathcal{R}^{m \times n}$, which is clearly finite-dimensional. It follows that we can write any element $K \in S$ as $K=$ $\sum_{i=1}^{d} r_{i} X_{i}$, where the matrices $\left\{X_{i}\right\} \subset \mathcal{R}^{m \times n}$ are a basis for $S$ when using the scalars $r_{i} \in \mathcal{R}$. For each $\alpha \in \mathbb{R}$, let $S_{\alpha}=\operatorname{span}\left\{X_{i}(\alpha)\right\}$.

Suppose $K \in S$. Then $K=\sum_{i=1}^{d} r_{i} X_{i}$ for some $r_{i} \in \mathcal{R}$. So $K(\alpha) \in S_{\alpha}$. Conversely, if $K \in \mathcal{R}^{m \times n}$ and $K(\alpha) \in S_{\alpha}$ for almost all $\alpha \in \mathbb{R}$, then there exists real numbers $r_{i}(\alpha)$ such that $K(\alpha)=\sum_{i=1}^{n} r_{i}(\alpha) X_{i}(\alpha)$. Applying Lemma 8, we conclude that there must exist rational functions $r_{i}$ that satisfy this equation, and thus $K \in S$.

Lemma 10. Suppose $G \in \mathcal{R}^{m_{2} \times n_{2}}$, and $S \subset \mathcal{R}^{n_{2} \times m_{2}}$ is an $\mathcal{R}$-subspace.

$S$ is $Q I$ with respect to $G \quad \Longleftrightarrow \quad h(S \cap M)=S \cap M$

Proof. A similar result appears in [5], and this one may be proved in a similar way. First use the equivalent characterization of Lemma 9, and then apply the matrix QI result to the matrices $G(\alpha)$ and subspaces $S_{\alpha}$ for almost all $\alpha \in \mathbb{R}$.

Lemma 11. Suppose $G \in \mathcal{R}^{m_{2} \times n_{2}}, S \subset \mathcal{R}^{n_{2} \times m_{2}}$ is an $\mathcal{R}$-subspace, and $W_{1}, W_{2}$ are projectors satisfying $W_{1} G W_{2}=G$. Then the two following statements are equivalent

i) $W_{2} S W_{1}$ is $Q I$ with respect to $G$

ii) $W_{2} h(S \cap M) W_{1}=W_{2}(S \cap M) W_{1}$
Proof. Note that for any $r \in \mathcal{R}$ and $K \in S, r W_{2} K W_{1}=$ $W_{2}(r K) W_{1} \in W_{2} S W_{1}$. Therefore $W_{2} S W_{1}$ is an $\mathcal{R}$ subspace. We may apply Theorem 10, and conclude that

$$
h\left(W_{2} S W_{1} \cap M\right)=W_{2} S W_{1} \cap M
$$

Using matrix identities, we find that for all $K \in M$,

$$
\begin{aligned}
W_{2} h(K) W_{1} & =-W_{2} K(I-G K)^{-1} W_{1} \\
& =-W_{2} K\left(I-W_{1} G W_{2} K\right)^{-1} W_{1} \\
& =-W_{2} K W_{1}\left(I-G W_{2} K W_{1}\right)^{-1} \\
& =h\left(W_{2} K W_{1}\right)
\end{aligned}
$$

We conclude that $K \in M \Longleftrightarrow W_{2} K W_{1} \in M$. Thus

$$
\begin{aligned}
W_{2} h(S \cap M) W_{1} & =h\left(W_{2} S W_{1} \cap M\right) \\
& =W_{2} S W_{1} \cap M \\
& =W_{2}(S \cap M) W_{1}
\end{aligned}
$$

All the steps above are reversible, so the converse holds as well, and we are done.

Lemma 12. Suppose $G \in \mathcal{R}^{m_{2} \times n_{2}}$. Let $W_{1}$ and $W_{2}$ be projectors.

$$
W_{1} G W_{2}=G \quad \Longleftrightarrow \quad\left\{\begin{array}{c}
\text { range } G \subset \text { range } W_{1} \\
\text { null } G \supset \text { null } W_{2}
\end{array}\right.
$$

Proof. Suppose range $G \subset$ range $W_{1}$. Let $x \in \mathcal{R}^{n_{2}}$. Now $G x \in$ range $G \subset$ range $W_{1}$. So there exists some $y \in \mathcal{R}^{n_{2}}$ such that $G x=W_{1} y$. Since $W_{1}$ is a projector, $W_{1} G x=W_{1}^{2} y=W_{1} y=G x$. Therefore, $W_{1} G=G$. Conversely, suppose $W_{1} G=G$. Then range $G \subset$ range $W_{1} G=$ range $W_{1}$. Similarly, we have $W_{2}^{T} G^{T} \Longleftrightarrow$ range $G^{T} \subset$ range $W_{2}^{T}$. The result follows from taking orthogonal complements and using rangenullspace duality.

Lemma 13. Suppose $P \in \mathcal{R}^{m \times n}, S \subset \mathcal{R}^{n_{2} \times m_{2}}$ is an $\mathcal{R}$-subspace, and $W_{1}, W_{2}$ are projectors satisfying

$$
\begin{aligned}
\text { range } W_{1} & =\text { range }\left[\begin{array}{ll}
P_{21} & P_{22}
\end{array}\right] \\
\text { null } W_{2} & =\text { null }\left[\begin{array}{l}
P_{12} \\
P_{22}
\end{array}\right]
\end{aligned}
$$

Then,

$$
\left[\begin{array}{cc}
I & 0 \\
0 & W_{1}
\end{array}\right] P\left[\begin{array}{cc}
I & 0 \\
0 & W_{2}
\end{array}\right]=P
$$

Proof. The range and nullspace requirement for $W_{1}$ and $W_{2}$ imply that

$$
\begin{gathered}
\text { range } P \subset \text { range }\left[\begin{array}{cc}
I & 0 \\
0 & W_{1}
\end{array}\right] \\
\text { null } P \supset \operatorname{null}\left[\begin{array}{cc}
I & 0 \\
0 & W_{2}
\end{array}\right]
\end{gathered}
$$


Applying Lemma 12, we conclude that

$$
\left[\begin{array}{cc}
I & 0 \\
0 & W_{1}
\end{array}\right] P\left[\begin{array}{cc}
I & 0 \\
0 & W_{2}
\end{array}\right]=P
$$

Proof of Theorem 5. We know from Lemma 2 that we can construct projectors $W_{1}$ and $W_{2}$ satisfying

$$
\begin{aligned}
\text { range } W_{1} & =\operatorname{range}\left[\begin{array}{ll}
P_{21} & P_{22}
\end{array}\right] \\
\text { null } W_{2} & =\text { null }\left[\begin{array}{l}
P_{12} \\
P_{22}
\end{array}\right]
\end{aligned}
$$

It follows by Lemma 13 that

$$
\left[\begin{array}{cc}
I & 0 \\
0 & W_{1}
\end{array}\right] P\left[\begin{array}{cc}
I & 0 \\
0 & W_{2}
\end{array}\right]=P
$$

In particular, we have $W_{1} P_{22} W_{2}=P_{22}, P_{12} W_{2}=P_{12}$, and $W_{1} P_{21}=P_{21}$. Now suppose that $S$ is IQI with respect to $P$. By definition, $W_{2} S W_{1}$ is QI with respect to $P_{22}$ and we may apply Lemma 11 and obtain

$$
W_{2} h(S \cap M) W_{1}=W_{2}(S \cap M) W_{1}
$$

Multiply by $P_{12}$ on the left and $P_{21}$ on the right and the result follows.

Proof of Theorem 7. Applying Theorem 5, we have $P_{12} h(S \cap M) P_{21}=P_{12}(S \cap M) P_{21}$. Restricting $S$ in the left-hand side to be proper yields $P_{12} h\left(S_{p}\right) P_{21} \subset$ $P_{12} S P_{21}$. Since the left-hand side is now a product of proper terms, it is itself proper. Therefore

$$
P_{12} h\left(S_{p}\right) P_{21} \subset\left(P_{12} S P_{21}\right) \cap \mathcal{R}_{p}^{m_{1} \times n_{1}}
$$

Now suppose $X \in P_{12} S P_{21}$ and $X$ is proper. By Theorem 5 , there is some $K \in S$ such that $P_{12} h(K) P_{21}$ is proper. By the proper-preserving property, $K$ must be proper. Therefore

$$
\left(P_{12} S P_{21}\right) \cap \mathcal{R}_{p}^{m_{1} \times n_{1}} \subset P_{12} h\left(S_{p}\right) P_{21}
$$

Equations (6) and (7) imply the sets are equal.

\section{Conclusion}

In this paper, we have characterized a class of decentralized control systems for which the set of achievable closed-loop maps is affine. This class, named internally quadratically invariant, includes all of the QI systems, as well as some which are not QI.

We also showed how to solve the associated minimumnorm controller synthesis problem, both for systems represented by rational functions and proper rational functions.

\section{Acknowledgements}

We would like to thank Hyung Sik Shin for some very helpful discussions.

\section{References}

[1] S. Boyd and C. Barratt. Linear controller design: limits of performance. Prentice-Hall, 1991.

[2] Y-C. Ho and K-C. Chu. Team decision theory and information structures in optimal control problems - Part I. IEEE Transactions on Automatic Control, 17(1):15-22, 1972.

[3] T. Kailath. Linear systems. Prentice-Hall Englewood Cliffs, NJ, 1980.

[4] L. Lessard and S. Lall. Reduction of decentralized control problems to tractable representations. In IEEE Conference on Decision and Control, pages 1621-1626, 2009.

[5] M. Rotkowitz and S. Lall. Decentralized control information structures preserved under feedback. In IEEE Conference on Decision and Control, pages 569-575, 2002.

[6] M. Rotkowitz and S. Lall. A characterization of convex problems in decentralized control. IEEE Transactions on Automatic Control, 51(2):274-286, 2006.

[7] H. S. Shin and S. Lall. Optimal decentralized control of linear systems via Groebner bases and variable elimination. To appear, American Control Conference, 2010.

[8] H. S. Witsenhausen. A counterexample in stochastic optimum control. SIAM Journal on Control, 6:131, 1968. 